\title{
What Does the DAP: IQ Measure?: Drawing Comparisons between Drawing Performance and Developmental Assessments
}

\author{
Gwendolyn Rehrig* $\quad$ Karin Stromswold ${ }^{\dagger}$
}

\begin{abstract}
Human figure drawing tasks like the Draw-A-Person (DAP) task have long been used to assess intelligence [9]. This study investigates the skills tapped by drawing and the risk factors associated with poor drawing. Self-portraits of 345 preschool children were scored by raters trained in using the DAP:IQ rubric [30]. Analyses of children's fine motor, gross motor, social, cognitive, and language skills revealed that only fine motor skill was an independent predictor of DAP:IQ scores. Being male and having a low birth weight were associated with lower DAP:IQ scores. These findings suggest that, although the DAP:IQ may not be a valid measure of cognitive ability, it may be a useful screening tool for fine motor disturbances in at-risk children, such as boys who were born at low birth weights. Furthermore, researchers who use human figure drawing tasks to measure intelligence should measure fine motor skill in addition to intelligence.
\end{abstract}

Keywords: DAP:IQ, intelligence, fine motor, developmental assessment

\section{Introduction}

When children draw, the images they produce differ from their real-world counterparts in appearance, even when they draw from a model [10]. From this, early researchers concluded that children's drawings reflect their world knowledge, and that drawing tasks could be used to measure children's intelligence $[9,10]$. Others have taken this finding to reflect the developmental course of cognitive flexibility: while children can integrate unique features into their drawings [19], younger children draw prototypical

This is an Accepted Manuscript of an article published by Taylor \& Francis Group in the Journal of Genetic Psychology on 12/01/2017, available online: https://www.tandfonline.com/doi/abs/10.1080/00221325. 2017.1392281

*Department of Psychology, University of California, Davis. Email: glrehrig@ucdavis.edu.

${ }^{\dagger}$ Department of Psychology and Center for Cognitive Science, Rutgers Unversity-New Brunswick versions of model objects because they lack the cognitive flexibility to integrate parts of the object into the silhouette of the whole object $[6,29,34,20]$.

[29] tested 4- to 6-year-old children in a drawing task and found that the 6-year-olds were better at drawing a saucepan as seen from an atypical viewing position than 4-year-olds, but the 4-years-olds improved when given less depth information (2D or $2 \& \frac{1}{2} \mathrm{D}$ model). [34] showed that 5-year-olds can integrate unique features of a model object into their drawings, but do so with greater difficulty than 8year-olds. Similarly, [6] showed that 6-year-olds were more likely to draw a prototypical version of a mug (depicting the handle even when hidden from view) when linguistic labels were used in the instructions to describe the object, but were able to accurately draw the mug from their viewpoint (handle occluded) when the object was not named. [20] assessed the development of realistic contours in human figure drawing. 
In a series of studies, 5- to 11-year-old children drew human figures, and their abilities to modify and integrate shapes in the countour of the figure were measured. The authors found that these qualities did not improve linearly with age, suggesting that multiple faculties — not just motor skill—are involved in the emergence of realistic human figure contours. [19] tested 7- to 12-year-olds on drawing a man, a woman, and a mug. Drawing improved in the older age groups for girls more so than boys, and boys focused on the outline of the object rather than including detail internal to the object. In all of these studies, the authors posit that children's drawings in one way or another tap world knowledge about the subject, and the authors use said drawings to make inferences about children's cognitive abilities.

Another body of evidence supporting the use of drawing tasks to measure intelligence is that human figure drawing tasks have been validated against standardized measures of full scale intelligence. For example, [1] have found relationships between full scale intelligence measures (Wechsler Intelligence Scale for Children-Revised [WISC-R], [39]; Wechsler Intelligence Scale for Children [WISC-III], [36]) and human figure drawing intelligence tasks designed by $[11,26,3]$. The DAP:IQ [30] has been normed against two full scale intelligence measures: the WISC-III [36] and the Reynolds Intellectual Assessment Scales (RIAS; [31]. [40] examined the reliability of the DAP:IQ [30] and found that reliability was high, but cautioned that some of the scoring criteria were more ambiguous than others, resulting in lower inter-rater reliability than reported by the test developers ${ }^{1}$. Reliability of the DAP:IQ may be negatively affected by fatigue when the task is completed repeatedly [21]. Recently, a large twin study [2] found that drawing performance and intelligence are both heritable, and found a relationship between DAP task performance at age four and intelligence

\footnotetext{
${ }^{1}$ Inter-rater reliability on the DAP:IQ reported by [40] was $r=0.83$, whereas the test developers [30] report inter-rater reliability measures of $r$ $=.95$ for drawings by older children and adults (age range 11-75), and $r=$ .91 for younger children (age range 6-11)
}

at age fourteen, ten years later.

Although human figure drawing tasks continue to be used to measure intelligence, the validity of these tasks as measures of intelligence has been challenged [14, 25]. [25] criticized the use of human figure drawing tasks as measures of intelligence citing inconsistent, low relationships between human figure drawing tasks of intelligence and standardized measures of intelligence, and the poor ability of drawing tasks to predict academic performance. They further suggest that ease of administration of human figure drawing tasks may be the only argument for their use, though they argue that this is not enough to compensate for poor task validity. These criticisms sparked a raging debate between researchers defending human figure drawing tasks as measures of IQ [4, $13,27]$ and researchers opposing their use [12, 15, 16, 24]. The controversy, however, did not halt the use of human figure drawing assessments by researchers. Indeed, in some studies DAP ${ }^{2}$ tasks have been used as the sole measure of intelligence where intelligence is a key study variable (e.g., [8]).

In recent years this debate has been revived by [14], who cite additional concerns about using human figure drawing tasks to measure intelligence. [14] compared the performance of four- and five-year-old children on the DAP:IQ to the children's performance on the Wechsler Preschool and Primary Scale of Intelligence ([WPPSI-III], [38]) and the performance of adults on the DAP:IQ and the Wechsler Abbreviated Scale of Intelligence Full scale IQ Two-Subtest ([WASI FSIQ-2], [37]). For children, [14] found a correlation between DAP:IQ scores and WPPSI-III performance; however, when DAP:IQ scores were compared with performance on individual subtests of the WPPSIIII, a significant correlation was found only for the Coding subtask-a nonverbal task that involves copying shapes. In

${ }^{2} D A P$ is used here to refer to any Draw-A-Person task with a scoring system designed to convert drawing scores into IQ scores or mental age equivalents. This includes the DAP QSS [26], the DAP:IQ [30], and the Goodenough-Harris drawing test [11]. 
addition, [14] found that the DAP:IQ had high false positive rates and high false negative rates as a screening test for identifying low functioning children and gifted children. DAP:IQ scores fared even less well as a measure of adults' IQ: adults' DAP:IQ scores were not significantly correlated with WASI full scale IQ scores and the DAP:IQ performed poorly as a screening test for identifying low-functioning or gifted adults.

Because the studies cited above only compared DAP performance with IQ, even those that do find a significant correlation between full-scale IQ and DAP:IQ cannot determine the extent to which cognitive ability is a predictor of DAP:IQ scores independent of other motor skill. A notable exception is a study by [32].

In this study, [32] assessed the cognitive, motor, and drawing ability at age five (as measured by performance on the Draw-A-Person: A Quantitative Scoring System [DAP:QSS], [26], another DAP task designed to assess intelligence), of 5-year-old children who were born very preterm (gestational age at birth $<32$ weeks). In this study, cognitive development was assessed using the Revised Amsterdam Child Intelligence Test ([RAKIT], [5]); and motor development was assessed using the Motor Assessment Battery for Children ([M-ABC], [33]), which collapses gross and fine motor development into a measure of overall motor development. A combined measure of cognitive and motor development, along with risk factors for delayed development at birth, were then compared with DAP performance to determine the relative contributions of each to drawing ability at age 5. The combined measure of cognitive and motor development was found to predict drawing performance, but having multiple risk factors at birth was not. Three limitations of [32]'s study are that they did not assess the role of age or sex in their model, nor did they assess the relative contributions of fine motor and gross motor skills independent of one another, or assess other areas that have been argued to be associated with drawing ability.

However, some researchers have argued that children's drawing skills are associated with socioemotional (e.g., [28, 17]), or cognitive and fine motor skills (e.g., [32]), or are associated with fine motor skills, but are indirectly mediated by verbal skills (e.g., [35]). The current study speaks to these claims by comparing drawing performance with fine motor, gross motor, language, social, and cognitive performance, and by including age and sex of the child in all analyses.

\section{Methods}

\subsection{Participants.}

The participants were 345 four- and-five-year olds who participated in a larger twin study. The study was approved by the Rutgers University Institutional Review Board, and was carried out in accordance with the Declaration of Helsinki. Parental consent was obtained in writing prior to data collection. By parent report, $49 \%$ of the participants were born low birth weight $(<2500 \mathrm{~g})$ with a mean birth weight of 2444 grams, and $57 \%$ of participants were born premature (gestational age at birth $<37$ weeks) with a mean gestational age at birth of 35.5 weeks (see Tables 1 and 2). $40.9 \%$ of boys in the sample were born both premature and low birth weight. Age at testing was calculated using each child's due date, not birth date, to correct for prematurity (henceforth called GA-corrected age).

\subsection{DAP:IQ Scores}

DAP:IQ raw scores were determined by four experimenters using the DAP:IQ scoring rubric [30], with possible raw scores ranging from 0-49. DAP:IQ raw scores were converted to standard scores using GA- corrected age at testing. The resulting DAP:IQ scores ranged from 51144 , with very high inter-rater reliability for 300 randomly 
TABle 1: Participant Demographics

\begin{tabular}{lr}
\hline & $\%$ of Sample \\
\hline Sex (\% male) & $51 \%$ \\
Twins & $94.2 \%$ \\
$\quad$ Monozygotic & $33.9 \%$ \\
$\quad$ Dizygotic & $60.3 \%$ \\
Mother's education level & \\
$\quad$ High school graduate & $1.2 \%$ \\
$\quad$ Some college or technical school & $14.8 \%$ \\
$\quad$ College graduate (B.A. or B.S.) & $46.4 \%$ \\
$\quad$ Advanced degree (M.A., Ph.D., or M.D.) & $37.1 \%$ \\
Ethnicity (\% non-Hispanic) & $94.5 \%$ \\
Race (\% Caucasian) & $95.4 \%$ \\
Annual household income & \\
$\quad$ Less than \$50,000 & $12.2 \%$ \\
Between \$50,000 and \$100,000 & $49.3 \%$ \\
$\quad$ Over \$100,000 & $33.9 \%$ \\
\hline
\end{tabular}

TABLE 2: Descriptive Statistics

\begin{tabular}{rrrrrrr}
\hline & $\begin{array}{l}\text { All Children } \\
(N=345)\end{array}$ & & \multicolumn{2}{l}{$\begin{array}{l}\text { Girls } \\
(N=169)\end{array}$} & \multicolumn{3}{l}{$\begin{array}{l}\text { Boys } \\
(N=176)\end{array}$} \\
\hline & $M$ & $S D$ & $M$ & $S D$ & $M$ & $S D$ \\
\hline DAP:IQ Score & 98.58 & 15.01 & 102.79 & 13.52 & 94.55 & 15.30 \\
Age at testing (months) & 60.80 & 6.02 & 60.42 & 5.86 & 61.16 & 6.17 \\
Gestational age at birth (weeks) & 35.50 & 3.05 & 35.77 & 2.66 & 35.24 & 3.37 \\
Birth weight (g) & 2443.50 & 651.93 & 2450.19 & 607.11 & 2437.08 & 693.92 \\
\hline
\end{tabular}

selected drawings $(r(298)=0.94, p<.0001)$.

\subsection{Developmental Assessments}

Two types of developmental assessments were used. First, children's developmental skills were assessed using Ages \& Stages Questionnaire (ASQ) scores [7]). The ASQ assesses children's fine motor, gross motor, language, social, and cognitive abilities ${ }^{3}$. ASQ scores for each age category (48 months -53.99 months, 54 months -59.99 months, and 60

${ }^{3}$ Cognitive ability is used to refer to performance on the problem solving portion of the ASQ, and language ability to refer to the communication portion of the ASQ. months) were transformed into z-scores so that comparisons could be made across age categories. Second, parents reported whether, within the most recent year, their child received therapeutic intervention that targeted any of the 5 developmental areas covered by the ASQ. The second assessment, which we view as a measure of a professional assessment indicating a problem at the time the DAP:IQ test was administered, will henceforth be referred to as therapy. For example, having received occupational therapy within the past year reflects evaluation by a professional occupational therapist and indicates fine motor problems. Similarly, receiving physical therapy was a proxy for gross 


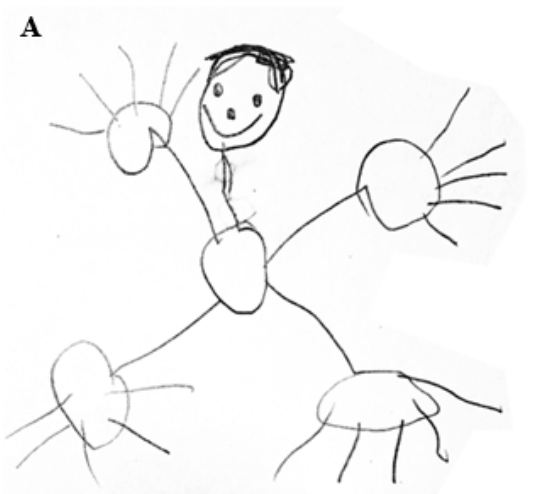

Age: 68.68 months DAP IQ: 106

C

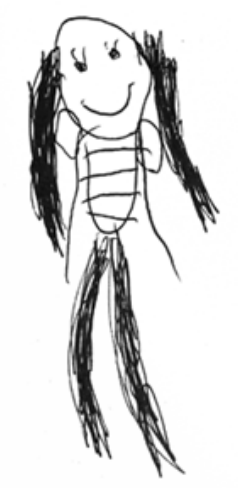

Age: 66.48 months

DAP IQ: 96

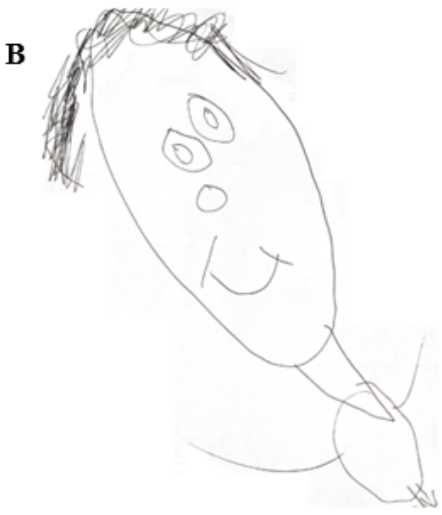

Age: 59.76 months

DAP IQ: 116

D

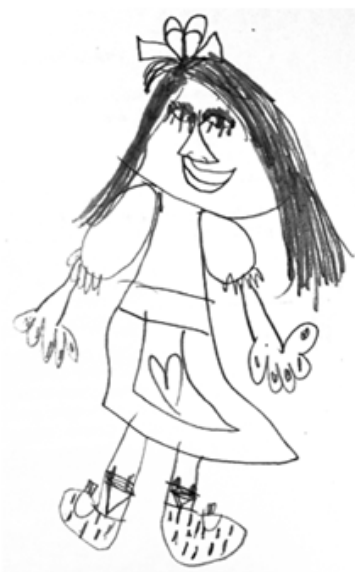

Age: 66.96 months

DAP IQ: 144

FIGURE 1: Drawings created by children in this study.

motor problems, receiving speech language therapy was a proxy for language problems, receiving behavioral therapy was a proxy for social/emotional problems, and receiving educational interventions (e.g., services from a reading or math specialist, full or part-time placement in a special classroom) was a proxy for cognitive delays.

\subsection{Procedure}

Questionnaires were sent to parents by mail, and were completed in the home. Included with the questionnaires were an age appropriate version of the ASQ and a Draw-APerson task drawing form. Parents followed instructions included with the ASQ to administer each assessment. For the DAP, children were given a drawing form and were instructed to draw a realistic self-portrait depicting the entire figure as seen from the front (see Figure 1 for representative drawings). ${ }^{4}$ Parents reported whether children had received any of several types of therapy (see Developmental assessments) within the most recent year as part of the study questionnaire. [30]:

${ }^{4}$ Exact instructions were as follows, taken from the DAP:IQ test manual I want you to draw a picture of yourself. Be sure to draw your whole body, not just your head, and draw how you look from the front, not the side. Do not draw a cartoon or stick figure. Draw the very best picture of yourself that you can. Take your time and work carefully. Go ahead. (p. 5) 


\subsection{Analysis}

\subsubsection{Correlation}

A bivariate correlational analysis was conducted to determine the relationship between DAP:IQ scores and GAcorrected age.

\subsubsection{Multiple Regression}

To determine what factors predict DAP:IQ performance, multiple regression analyses were conducted. In all regression models, DAP:IQ scores were the dependent variable, and age and sex were included as independent predictors. Three models were constructed: one in which birth weight and GA-corrected age were included as independent predictors, and two in which developmental assessments (therapy or Z-transformed ASQ scores) were included as independent predictors.

\section{Results}

GA-corrected age was marginally correlated with DAP:IQ scores $(r(343)=.12, p=.02$, alpha criterion $=.01$; see Figure 2). This finding, though marginal for our alpha, is nonetheless surprising. According to [30], converting DAP:IQ raw scores to standard scores should have corrected for age.

\subsection{Demographic Analyses}

\subsubsection{Sex}

Although the mean DAP:IQ scores for girls and boys were both in the normal range, girls' DAP:IQ scores were on average 8.2 points higher than boys' $(F(1,343)=28.02, p<$ .0001 ; see Figure 3 and Table 2).

\subsubsection{Prematurity and Birth Weight}

For DAP:IQ scores, multiple regression analyses with birth weight and gestational age as independent variables revealed that higher birth weight was a marginally significant predictor of higher DAP:IQ scores for boys $(\beta=.31, p<.05)$, but neither birth weight or gestational age even marginally predicted DAP:IQ scores for girls (Table 3). Neither birth weight or gestational age at birth influenced DAP:IQ scores when boys' and girls' data were combined.

\subsection{Developmental Skills}

Multiple regression analyses with the 5 ASQ scores, sex, and age as independent variables revealed that higher fine motor ASQ scores $(\beta=.43, p<.0001)$ and female sex $(\beta$ $=.17, p=.0004)$ were significant independent predictors of higher DAP:IQ scores (see Table 4). When skill-specific therapies were used as proxies for the 5 developmental areas, female sex $(\beta=.27, p<.0001)$, age $(\beta=.14, p=.006)$, and not having received fine motor (occupational) therapy ( $\beta=-.23, p=.002$ ) were significant predictors of higher DAP:IQ scores. Surprisingly, having received gross motor (physical) therapy was marginally associated with higher DAP:IQ scores $(\beta=.14, p=.03)$.

\section{Discussion}

To recap, birth weight was a marginally significant independent predictor of boys' DAP:IQ scores, but not girls' DAP scores. These results suggest that perinatal risk factors are associated with poor figure drawing in boys, but not girls. In addition, older age, female sex, and higher measures of fine motor skills were independent predictors of higher DAP:IQ scores. Importantly, higher measures of cognitive ability did not predict DAP:IQ scores.

Consistent with [32]'s finding, in the current study, females outperformed males on the DAP:IQ. Although we 


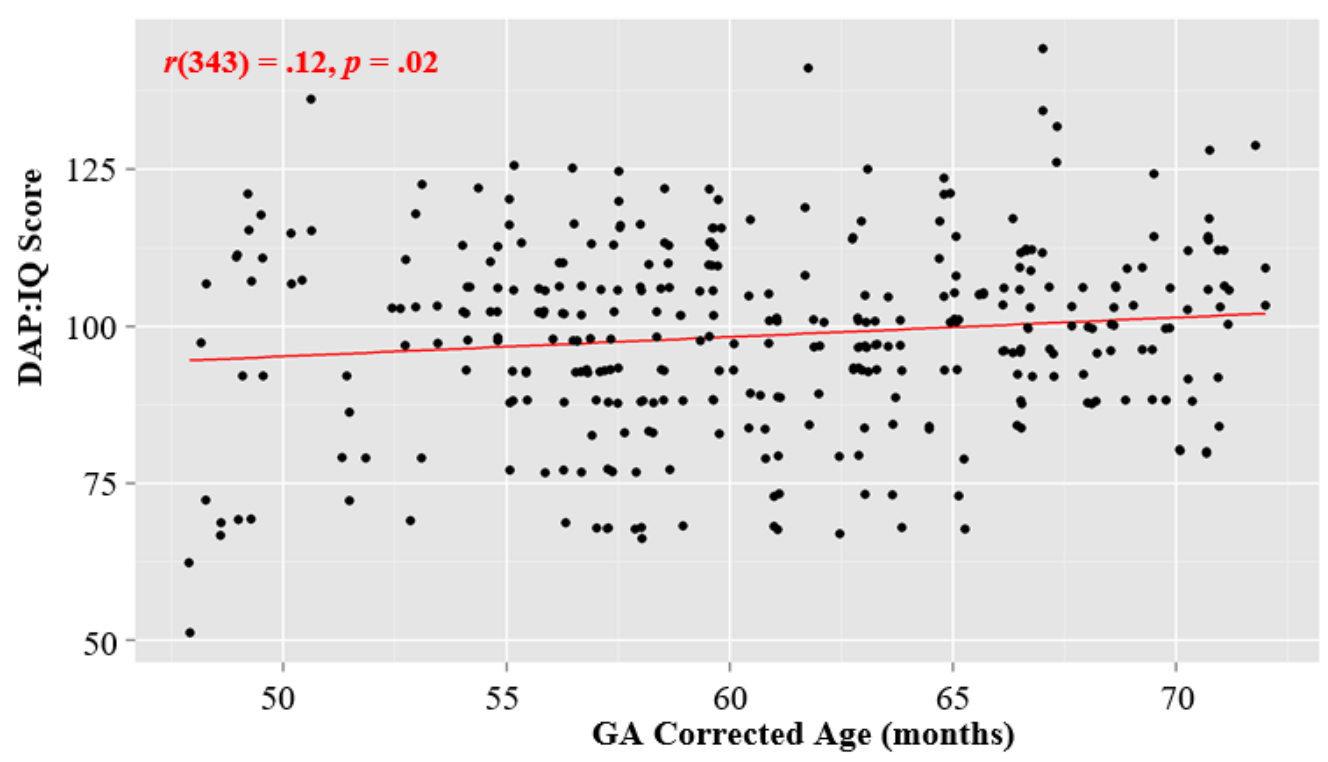

FIGURE 2: Scatterplot comparing DAP:IQ scores with gestational age (GA) corrected age in months.

TABLE 3: Multiple Regression Analysis for DAP:IQ and Birth Demographics

\begin{tabular}{rrrrrrr}
\hline & $\begin{array}{l}\text { All Children } \\
(N=345)\end{array}$ & \multicolumn{2}{l}{$\begin{array}{l}\text { Girls } \\
(N=169)\end{array}$} & \multicolumn{2}{l}{$\begin{array}{l}\text { Boys } \\
(N=176)\end{array}$} \\
\hline & $\beta$ & $p$ & $\beta$ & $p$ & $\beta$ & $p$ \\
\hline Age at testing & .14 & .007 & .07 & $\mathrm{~ns}$ & .20 & .006 \\
Sex & .29 & $<.0001$ & - & - & - & - \\
Birth Weight & .13 & $\mathrm{~ns}$ & -.002 & $\mathrm{~ns}$ & .31 & .04 \\
Gestational Age at Birth & -.12 & $\mathrm{~ns}$ & -.14 & $\mathrm{~ns}$ & -.19 & $\mathrm{~ns}$ \\
\hline
\end{tabular}

found that low birth weight was a risk factor for low DAP scores in boys (but not girls), contrary to [32], prematurity was not an independent predictor of either boys' or girls' DAP:IQ scores in the current study. [32]. did not assess the potential contribution of age even though [32]'s preterm children were chronologically 4 months younger (and gestationally 7 months younger) than their full term children. It is well known that children's figure drawing improves rapidly during early childhood and, consistent with this, we found that a child's age was a strong predictor of drawing performance. We believe that [32]'s "prematurity" effect is instead an age effect. [32]'s findings also suffer from omitting sex as a predictor, despite finding significant sex differences in a separate analysis, and despite known sex differences in drawing ability for young children (see [18]). We found no positive relationship between cognitive development or gross motor skills and DAP performance, while [32]. found that a combined measure of cognitive and motor development predicted drawing ability. This may have occurred because the independent contribution of fine motor development was not assessed by the M-ABC (Movement Assessment Battery for Children [M-ABC], [33]), and the contribution of fine motor development may have been attenuated by the gross motor portions of the assessments.

DAP:IQ Measures Fine Motor, Not Cognitive Skills. For developmental assessments, DAP:IQ scores were related to 
TABLE 4: Multiple Regression Analyses comparing DAP:IQ Scores and Developmental Assessments

\begin{tabular}{rlccc}
\hline & $\begin{array}{l}\text { ASQ scores } \\
(N=345)\end{array}$ & $\begin{array}{l}\text { Therapy } \\
(N=345)\end{array}$ & \\
\hline & $\beta$ & $p$ & $\beta$ & $p$ \\
\hline Age at Testing & .09 & .05 & .14 & .006 \\
Sex & .17 & .0004 & .27 & $<.0001$ \\
Fine Motor & .43 & $<.0001$ & -.23 & .002 \\
Gross Motor & -.10 & $\mathrm{~ns}$ & .14 & .03 \\
Language & .07 & $\mathrm{~ns}$ & -.03 & $\mathrm{~ns}$ \\
Cognitive & .06 & $\mathrm{~ns}$ & -.0007 & $\mathrm{~ns}$ \\
Social & -.03 & $\mathrm{~ns}$ & -.02 & $\mathrm{~ns}$ \\
\hline & & & &
\end{tabular}

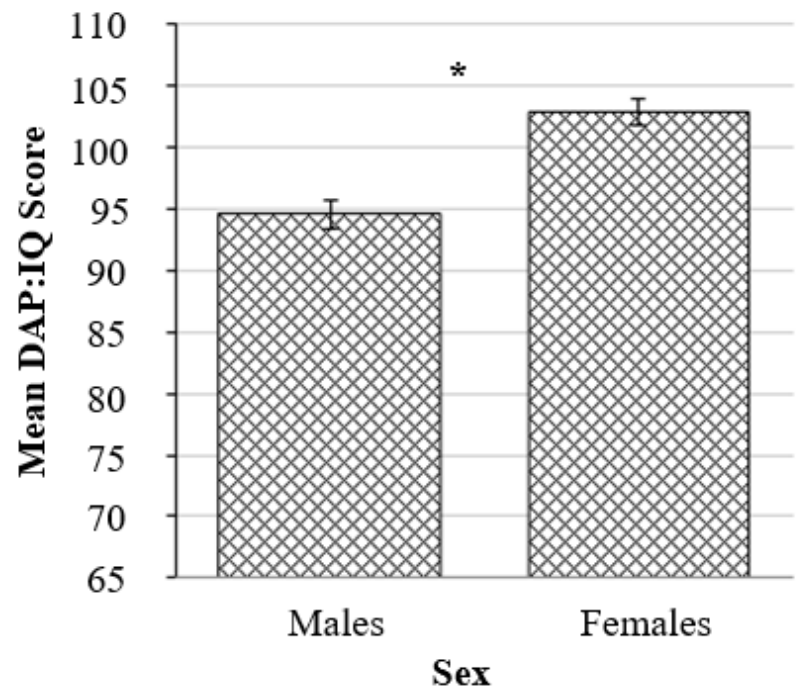

FIGURE 3: Mean DAP:IQ scores for males and females. Error bars indicate standard error of the mean. The asterisk $\left(^{*}\right)$ indicates significance $(p<.0001)$.

fine motor ability and not cognitive ability. Why, then, have some reported that DAP scores are good measures of non-verbal IQ? It is possible that cognitive ability and drawing ability develop in parallel, in which case previous research may have found an association between human figure drawing and intelligence because other abilities were not included in the comparison. ${ }^{5}$ The likelihood of this explanation can be illustrated using our data: when a multiple regression analysis was conducted with DAP:IQ

${ }^{5}$ See [22] for a similar argument regarding an illusory relationship between the development of drawing ability and theory of mind development. as the dependent variable and age, sex, and ASQ cognitive scores as the only independent variables, higher cognitive ability strongly predicted higher DAP:IQ scores $(\beta=.21, p<$ $.0001)$. However, when the same analysis was repeated with the addition of ASQ fine motor scores as an independent variable, the predictive contribution of cognitive ability disappeared entirely $(\beta=.04, p=.44)^{6}$. It is possible that studies which have compared intelligence and drawing without administering a fine motor assessment (such as [2]) would show similar trends had the contribution of fine motor skill been included.

Another possibility raised by [14] is that portions of the IQ tasks administered by [30] and the DAP:IQ task capture similar abilities, but the relationship between full scale IQ scores and DAP:IQ scores are nonexistent, or weak. Imuta et al. only found a relationship between DAP:IQ scores and the WPPSI-III Coding subtest which includes identifying and drawing shapes [38]. This is unsurprising given that both the DAP:IQ and the WPPSI-III Coding subtest involve drawing.

Limitations of the Current Study. All the measures in this study relied on parental report, which is unlikely to

${ }^{6}$ This is not to say that there are no cognitive aspects of drawing, or that drawings cannot be used to assess any aspect of cognition. Rather, it indicates that human figure drawing tasks like the DAP that are designed to measure general intelligence are not primarily tapping cognitive ability, despite claims to the contrary. 
be objective. In future work along this line of research, standardized assessments carried out by professionals should be used to ascertain children's developmental skills. We found that ASQ fine motor scores strongly predicted DAP:IQ scores. In many ways, this is unsurprising. The ASQ fine motor assessment includes tasks that require the use of a writing implement either to draw or to write. To assess the contribution of fine motor skills to drawing performance independent of the skills needed to use a writing implement, future work should use assessments that measure fine motor skills by performance on tasks of manipulation alone. Another limitation of the current study is that we did not measure working memory span, which has been implicated in drawing performance for children of this age group [23]. Finally, the analyses used in the current study could not capture indirect effects of one variable on another. If drawing ability is mediated by cognitive ability, our analyses would not detect that relationship. Future research should also consider the indirect effects one skill may have on another (for example, [35]).

Taken as a whole, the results of this study suggest that DAP:IQ scores are a robust and reliable indicator of fine motor ability, but not cognitive ability. Given the ease of administration and reliability of the DAP:IQ, our results support the use of the DAP:IQ as a fine motor screening tool for high risk children (e.g., low birth weight boys) whose fine motor skills should be formally assessed. Our results also suggest that future attempts to develop human figure drawing tests of intelligence must include assessments of a broad range of skills, and not just cognitive skills. Finally, to avoid the problem of circularity, future research should employ standardized assessments of motor skills that do not involve coordination of a writing implement.

\section{Acknowledgments}

We thank Jacob Feldman, Kimberly Brenneman, and two anonymous reviewers for their helpful feedback, which greatly improved the manuscript. This work was made possible by the efforts of our research assistants, whom we thank for their many hours of hard work: Carine Abraham, Chandni Patel, Alnida Espinosa, Gabriela Bess, and Melinh Lai.

\section{Funding}

This work was supported with funding from the National Science Foundation for the Social, Behavioral, and Economic Sciences (SBE BCS-0002010, BCS-0042561, BCS0124095, and BCS-0446838) and the Integrative Graduate Education and Research Traineeship (DGE IGERT 0549115). The research was also generously supported by the Busch Biomedical Research Fund and the BamfordLahey Children's Foundation.

\section{References}

[1] S. C. Abell, W. Wood, and S. J. Liebman. Children's human figure drawings as measures of intelligence: the comparative validity of three scoring systems. Journal of Psychoeducational Assessment, 19(3):204-215, 2001.

[2] R. Arden, M. Trzaskowski, V. Garfield, and R. Plomin. Genes influence young children's human figure drawing and their association with intelligence a decade later. Psychological Science, 25(10):1843$1850,2014$.

[3] A. J. Ayres and W. Reid. The self-drawing as an expression of perceptual-motor dysfunction. Cortex, 2(2):254-265, 1966. 
[4] A. N. Bardos. Human figure drawings: abusing the abused. School Psychology Quarterly, 8(3):177-181, 1993.

[5] N. Bleichrodt, P. J. Drenth, J. N. Zaal, and W. C. Resing. Revision Amsterdam Child Intelligence Test. Swets \& Zeitlinger, Lisse, 1984.

[6] J. G. Bremner and S. Moore. Prior visual inspection and object naming: two factors that enhance hidden feature inclusion in young children's drawings. British Journal of Developmental Psychology, 2(4):371-376, 1984.

[7] D. D. Bricker, J. Squires, and L. Mounts. Ages \& Stages Questionnaires: A Parent-Completed, ChildMonitoring System. Paul H. Brookes, Baltimore, MD, 1999.

[8] O. Ezenwosu, I. Emodi, A. Ikefuna, and B. Chukwu. Academic performance and intelligence scores of primary school-aged children with sickle cell anemia. Pediatric Hematology and Oncology, 30:733-741, 2013.

[9] F. Goodenough. Measurement of Intelligence by Drawings. World Book Co, Oxford, England, 1926.

[10] F. Goodenough. Studies in the psychology of children's drawings. Psychological Bulletin, 25(5):272, 1928.

[11] F. Goodenough and D. B. Harris. The GoodenoughHarris Drawing Test. Harcourt, Brace, and World, New York, 1963.

[12] F. M. Gresham. What's wrong in this picture? School Psychology Quarterly, 8(3):182-186, 1993.

[13] W. H. Holtzman. An unjustified, sweeping indictment by motta et al. of human figure drawings for assessing psychological functioning. School Psychology Quarterly, 8(3):189-190, 1993.
[14] K. Imuta, D. Scarf, H. Pharo, and H. Hayne. Drawing a close to the use of human figure drawings as a projective measure of intelligence. PLoS ONE, 8(3):e58991, 2013

[15] R. W. Kamphaus. Comment on "the use and abuse of human figure drawings". School Psychology Quarterly, 8(3):187-188, 1993.

[16] H. M. Knoff. The utility of human figure drawings in personality and intellectual assessment: why ask why? School Psychology Quarterly, 8(3):191-196, 1993.

[17] J. ter Laak, M. de Goede, A. Aleva, and P. van Rijswijk. The draw-a-person test: an indicator of children's cognitive and socioemotional adaptation? The Journal of Genetic Psychology, 166(1):77-93, 2005.

[18] C. Lange-Küttner. Sex differences as a statistical variable. In A Alvinius, editor, Gender Differences in Different Contexts, pages 3-20. InTech Open Press, Rijeka, 2017.

[19] C. Lange-Küttner. Sex differences in visual realism in drawings of animate and inanimate objects. Perceptual and Motor Skills, 113(2):439-453, 2011.

[20] C. Lange-Küttner, A. Kerzmann, and J. Heckhausen. The emergence of visually realistic contour in the drawing of the human figure. British Journal of Developmental Psychology, 20:439-463, 2002.

[21] C. Lange-Küttner, E. Küttner, and M. Chromekova. Deterioration and recovery of dap iq scores in the repeated assessment of the naglieri draw-a-person (dap) test in 6- to 12-year-old children. Psychological Assessment, 26(1):297-306, 2014.

[22] A. Leslie and L. Thaiss. Domain specificity in conceptual development: neuropsychological evidence from autism. Cognition, 43:225-251, 1992. 
[23] S. Morra and S. Panesi. From scribbling to drawing: the role of working memory. Cognitive Development, 43:142-158, 2017.

[24] R. W. Motta, S. G. Little, and M. I. Tobin. A picture is worth less than a thousand words: response to reviewers. School Psychology Quarterly, 8(3):197-199, 1993.

[25] R. W. Motta, S. G. Little, and M. I. Tobin. The use and abuse of human figure drawings. School Psychology Quarterly, 8(3):162-169, 1993.

[26] J. A. Naglieri. Draw A Person: A Quantitative Scoring System. Psychological Corporation, San Antonio, Texas, 1988.

[27] J. A. Naglieri. Human figure drawings in perspective. School Psychology Quarterly, 8(3):170-176, 1993.

[28] J. A. Naglieri, T. J. MacNeish, and A. Bardos. Draw a person: screening procedure for emotional disturbance; dap: sped. In Austin. Pro-Ed, Texas, 1991.

[29] D. Picard and K. Durand. Are young children's drawings canonically based? Journal of Experimental Child Psychology, 90:48-64, 2005.

[30] C. R. Reynolds and J. A. Hickman. Draw-a-person intellectual ability test for children, adolescents, and adults: examiner's manual. In Austin. Pro-Ed, Texas, 2004.

[31] C. R. Reynolds and R. W. Kamphaus. Reynolds Intellectual Assessment Scales. Psychological Assessment Resources, Lutz, Florida, 2003.

[32] S. Schepers, M. Deković, and M. Feltze. Drawings of very preterm-born children at 5 years of age: a first impression of cognitive and motor development? European Journal of Pediatrics, 171:43-50, 2012.
[33] B. M. Smits-Engelsman. Movement ABC; Dutch Guide. Swets and Zeitlinger, Lisse, 1992.

[34] M. Taylor and V. R. Bacharach. Constrains on the visual accuracy of drawings produced by young children. Journal of Experimental Child Psychology, 34:311-329, 1982.

[35] A. Toomela. Drawing as a verbally mediated activity: a study of relationships between verbal, motor, and visuospatial skills and drawing in children. International Journal of Behavioral Development, 26(3):234-247, 2002.

[36] D. Wechsler. The Wechsler Intelligence Scale for Children (third ed.) The Psychological Corporation, San Antonio, Texas, 1991.

[37] D. Wechsler. Wechsler Abbreviated Scale of Intelligence. The Psychological Corporation, San Antonio, Texas, 1999.

[38] D. Wechsler. Wechsler Preschool and Primary Scale of Intelligence (Third ed.) The Psychological Corporation, San Antonio, Texas, 2002.

[39] D. Weschler. Weschler Intelligence Scale for Children-Revised. The Psychological Corporation, San Antonio, Texas, 1974.

[40] T. O. Williams, A. M. Fall, R. C. Eaves, and S. WoodsGroves. The reliability of scores for the draw-a-person intellectual ability test for children, adolescents, and adults. Journal of Psychoeducational Assessment, 24:137-144, 2006. 